Ricerca in Psicoterapia / Research in Psychotherapy 2010; 1(13): 92-119. http://www.researchinpsychotherapy.net

\title{
La ricerca di processo nel decennio 1998-2007: contributo a una review critica
}

\author{
Stefano Manzo ${ }^{1}$
}

Sommario: Questo studio preliminare è un primo contributo ad una revisione critica relativa alla ricerca di processo in psicoterapia. Lo scopo del lavoro mira a comprendere in quante forme si declina la ricerca di processo, come essa si diversifica, in ragione di quali dimensioni. Attraverso una griglia costruita ad hoc, sono stati segmentati gli studi di processo (sia di processo che di processo-esito) estrapolati da 13 riviste di settore relativi al decennio 1998-200\%. Gli obiettivi erano: a) quantificare e classificare la produzione scientifica relativa agli ultimi dieci anni; b) esplorare le principali modalità che ne diversificano e organizzano il campo. Sono emersi due modelli epistemologici: il modello d'analisi e quello interpretativo. Questi organizzano tre principali approcei alla ricerca sul processo che abbiamo definito: Approccio Congetturale, Sperimentale e Misto; questi sollevano differenti interrogativi riguardo alla significatività, alla scelta delle unità d'analisi e al rapporto tra oggetto della ricerca e metodologia adottata.

Parole chiave: review critica; ricerca sul processo; ricerca sullesito; ricerca sul processo-esito.

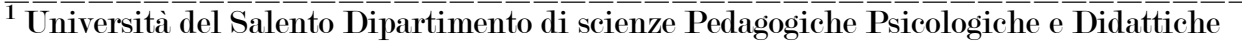

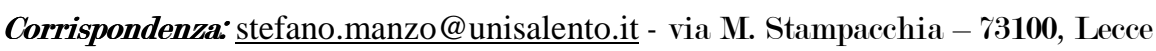

Ricevuto: 19 Novembre 2009 - Revisionato: 13 Aprile 2010 - Accettato: 22 Maggio 2010 
Ricerca in Psicoterapia / Research in Psychotherapy 2010; 1(13): 92-119. http://www.researchinpsychotherapy.net

\section{Introduzione}

La ricerca di processo si è caratterizzata sin dai suoi esordi per l’impiego di molteplici approcci, metodologie e strumenti. Questa eterogeneità appare il riflesso sia della complessità del suo oggetto di studio, sia di una frammentazione dovuta ad aspetti di ordine epistemologico, concettuale e metodologico (Kiesler, 1971; Garfield, 1990). Le principali motivazioni addotte riguardano l'eclettismo metodologico e la scissione tra ricerca e clinica.

Eclettismo metodologico. Frutto della sovrapposizione di differenti paradigmi a volte incommensurabili, oppure riflesso di una frammentarietà tra studi che convivono in una sorta di misconoscimento reciproco. Si pensi, ad esempio, alle problematiche metodologiche legate all'assenza di precise e condivise unità di analisi necessarie all’individuazione e segmentazione di specifici aspetti del processo (Kiesler, 1973; Russell \& Stiles, 1979; Greenberg, 1986; Greenberg \& Pinsof, 1986; Henry et al., 1986; Russell, 1988; Russell \& Staszewski, 1988; Elliott, 1991; Russell, 1994; Orlinsky et al., 2004) oppure all'uso di metodi aggregativi che analizzano il processo terapeutico come un'unica dimensione/proprietà. I metodi aggregativi, sono stati criticati in prima istanza da Kiesler $(1966,1973)$ in quanto fondati sul "mito dell'uniformità" [Elliott (1983); Rice \& Greenberg (1984); Greenberg (1986); Greenberg \& Pinsof (1986); Hilliard (1993); si veda anche Gelo e Auletta (2010) in questo numero] ${ }^{1}$, e in successive, ricerche [Gurman, 1973; Gottman \& Markman (1978) cit. in Rice \& Greenberg (1984)] che ne hanno dimostrato la debolezza metodologica, spingendo diversi studiosi all'esplorazione di nuovi paradigmi.

Soluzioni in questa direzione sono rappresentate dal paradigma del "change process research" (Rice \& Greenberg, 1984; Greenberg, 1991) o dal "treatment process" (Stiles, Shapiro, \& Elliott, 1986; Stricker \& Gold, 1993; Orlinksy et al., 2004).

Scissione tra ricerca e clinica. Sotto il concetto "ombrello" di ricerca di processo trovano spazio sia gli studi empirici rigorosi, sia gli studi di casi clinici. La scissione ${ }^{2}$ che attraversa il settore ha condotto a una

\footnotetext{
${ }^{1}$ Il mito dell'uniformità del processo terapentico giudica inadeguate quelle ricerche che analizzano il processo terapeutico come un fenomeno monodimensionale. Queste ricerche considerano implicitamente tutte le risposte del terapeuta o del paziente uguali dal punto di vista del loro impatto, queste hanno tutte le stesso peso. La conseguenza sul piano della metodologia conduce all'uso delle frequenze aggregate. Un tipico approccio, ad esempio, è caratterizzato dalla selezione (anche casuale) di campioni di unità d'analisi da una o più sedute, confrontando i campioni di una singola seduta, o, tra le sedute, con l'outcome. Un assunto sottostante agli approcei aggregativi è che il comportamento di un dato cliente o terapenta è considerato positivo o negativo indipendentemente dal contesto nel quale avviene. Ciò significa che vi è poca variabilità tra paziente terapenta e sedute (Kiesler, 1966) e che questi sono da considerarsi variabili indipendenti.

${ }^{2}$ Svariati sono i ricercatori e gli studi che, con diverse motivazioni, sottolineano questo gap (Luborsky, 1972; Morrow-Bradley, \& Elliott, 1986; Greenberg \& Pinsof, 1986; Goldfried \& Wolfe, 1996, 1998; Kopta, 1999; Pachankis \& Goldfried, 200\%), auspicano una maggiore generalizzabilità dei risultati della ricerca alla pratica clinica (Greenberg, 1986; Bentler et al., 1996; Campbell, 1996; Fensterheim \& Raw, 1997; Goldfried \& Wolfe, 1996; Strupp 1997) e direzionano i loro sforzi di ricerca verso l’individuazione di elementi di congiunzione tra i due settori.
} 
Ricerca in Psicoterapia / Research in Psychotherapy 2010; 1(13): 92-119. http://www.researchinpsychotherapy.net

pluralizzazione delle ricerche e spesso anche a un'incomparabilità dei risultati.

Una task force dell'American Psychological Association, (2005) ha espresso il bisogno di ridurre il gap clinica-ricerca includendo, nella sua definizione di evidence-based practice, l’integrazione delle migliori ricerche disponibili con la valutazione di clinici esperti. La Task Force ha proposto due criteri attraverso i quali ogni trattamento dovrebbe essere valutato: a) efficacia del trattamento e b) utilità clinica. Questa scelta trova una giustificazione concettuale e una empirica: storicamente l'area della ricerca in psicoterapia presentava un errore sistematico relativo alla prospettiva di osservazione, che secondo Orlinsky \& Howard (1978) è stata condotta dal vertice degli osservatori non partecipanti (i ricercatori). Gli eventi o le esperienze valutate da osservatori esterni o da giudici ottenevano una validità scientifica maggiore di quella ottenuta tramite le esperienze riportate o osservate dai partecipanti. Grazie all'introduzione di diversi strumenti (ad esempio, il Therapy Session Report di Orlinsky \& Howard o il Barret-Lennard's Relationship Inventory) e al successo ottenuto nel predire l'outcome, c'è stata una inversione di tendenza poiché si è rilevato che i self-report, se paragonati alle misure osservazionali, sono maggiormente predittivi dell'outcome [per una rassegna si veda Greenberg \& Pinsof (1986)].

Entro un panorama così vasto ed eterogeneo, il concetto di processo psicoterapeutico è andato progressivamente articolandosi così da perdere una qualificazione semantica unitaria (Dazzi, 2006; Lingiardi, 2006; Salvatore, 2007). Esso si presta a essere un costrutto "canestro" (Salvatore 2007) in grado di raccogliere in sé una miriade di definizioni e sfumature che, se non chiaramente distinte, possono divenire causa di confusione (Orlinsky et. al, 2004). Parte della letteratura, infatti, è concentrata sulla definizione e ridefinizione incessante del costrutto di processo riguardo alle scoperte empiriche e alle conseguenti riflessioni che ne amplificano l'alone semantico (Kiesler, 1973; Pinsof \& Greenberg, 1986; Marmar, 1990; Elliott, 1991; Hill, 1994, 2004; Orlinsky, 2004).

La review critica che promuoviamo - di cui questo lavoro costituisce uno studio preliminare - si propone di rilevare le principali "derive semantiche" che ha subito il concetto di processo, individuando quelle biforcazioni fonte di variabilità e analizzarne le cause. Accanto a una mappatura puntuale degli studi presenti in letteratura proponiamo unanalisi che evidenzi gli aspetti di convergenza e divergenza tra le diverse dimensioni indagate. Le domande che hanno guidato il presente lavoro sono state: come si fa ricerca di processo? In quanti modi? Quali sono i fattori comuni e le dimensioni latenti che organizzano tali modalità? L'obiettivo è consistito nell'individuare e descrivere $\mathrm{i}$ vettori organizzanti la ricerca di processo analizzando - attraverso un insieme di variabili - la diversa morfologia che essa assume.

Operativamente ciò si è tradotto nei seguenti obiettivi: 1) quantificare la produttività delle pubblicazioni riguardanti la process research e analizzarne i trend; 2) descrivere le diverse forme che la process research assume tramite una griglia costruita ad hoc, 3) interpretare e mappare le dimensioni sottostanti che ne organizzano le forme utilizzate. 
Ricerca in Psicoterapia / Research in Psychotherapy 2010; 1(13): 92-119. http://www.researchinpsychotherapy.net

\section{Metodo}

Le variabili utilizzate sono state organizzate entro due are d'indagine: cultura e strategie di ricerca. Prima di descriverle in dettaglio vorremmo precisare la definizione del concetto di processo che assumiamo a fondamento della nostra rassegna, ancorandoci a un insieme di ricerche che rappresentano punti di riferimento sufficientemente condivisi all'interno della letteratura (Kiesler, 1973; Strupp, 1973; Orlinsky \& Howard, 1978; Rice \& Greenberg, 1984; Greenberg, 1986; Greenberg \& Pinsof, 1986; Elliott, 1991; Stiles, 1988; Hill, 1994, 2004; Russell, 1994; Orlinsky et al., 2004). Con il concetto di processo ci riferiamo allo studio dell'interazione tra il sistema cliente e il sistema terapeuta; l'interazione riguarda sia ciò che avviene nella seduta, sia ciò che si sviluppa fuori della stessa (il pensare al terapeuta/cliente, l'eseguire compiti assegnati, etc.), sia gli incontri vis a vis in condizioni sperimentali, virtuali, etc. Con interazione stiamo contemplando tanto il processo quanto il suo esito immediato, a medio e a lungo termine.

Nell'area definita Cultura abbiamo inserito quelle variabili "trasversali" alla ricerca stessa, che non influenzano direttamente il processo. Quest'area comprende variabili legate alla provenienza istituzionale degli autori (clinici e ricercatori), la cultura o "humus" che ne orienta le prassi, l’influenza generata dalla rete cui appartengono; è prevalentemente in questarea che ricadono le problematiche riguardanti la scissione tra la clinica e la ricerca. Assumiamo che vi sia una relazione tra la tipologia di autore e la tipologia di studi prodotta e assumiamo che gli studi congiunti (ricercatori e clinici insieme) costituiscano un indice di coesione, per cui minore è il valore dell'indice maggiore il gap.

Utilizzeremo quattro variabili:

a) Tipologia di Autore. Descrive la provenienza istituzionale degli autori. Questa variabile ci informa se gli autori appartengono istituzionalmente ad ambiti accademici o clinici, oppure a entrambe le dimensioni. I dati si riferiscono congiuntamente al primo e al secondo autore. Le tipologie di autore sono: a1) Accademico; a2) Clinico; a3) Entrambi.

b) Area Geografica. Riguarda l'area geografica dell'istituzione alla quale l'autore della ricerca afferisce. L'area geografica è una variabile che può fornire informazioni trasversali sulle diverse tipologie di ricerche prodotte poiché influenzate dalle esigenze sociali, politiche e culturali che le organizzano. Le aree geografiche sono: b1) Africa; b2) Asia (comprendente Israele); b3) Australia; b4) Canada; b5) Europa; b6) Sud America; br) USA.

c) Area Psicoterapica di Intervento. Riguarda le modalità di trattamento impiegate. E prevalentemente attraverso questa variabile che emergono problematiche relative a potenziali competizioni tra modelli/scuole. Quest’area è di pertinenza del clinico e del suo cliente ed è strettamente legata all'esercizio dell'intervento. Le variabili che considereremo sono: c1) Cognitiva: comprende le terapie costruttiviste, cognitive e cognitivocomportamentali. (2) Ermeneutica: comprende le terapie esperienziali e fenomenologiche. c3) PDT: comprende le terapie psicoanalitiche e 
Ricerca in Psicoterapia / Research in Psychotherapy 2010; 1(13): 92-119. http://www.researchinpsychotherapy.net

psicodinamiche. c4) Mista: comprende tutte le terapie che integrano approcei differenti, multidisciplinari (ad esempio, sistemico- familiare). c5) Multipla: comprende le terapie utilizzate ai fini di un confronto ad esempio, per individuare la terapia d'elezione (cognitivo vS comportamentale). e6) Non Specificata: comprende quegli studi in cui non viene esplicitato il modello di intervento. c7) Altro: comprende la terapia familiare, quella comportamentale e quella eclettica.

d) Resoconto. Riguarda la modalità di resocontazione del caso clinico. Questa variabile è informativa di più aspetti: ad esempio, implicazioni epistemologiche (relative al differente significato che assume un certo tipo di ricostruzione del processo), implicazioni sul piano clinico (invasività, pericolosità o legittimità di uno strumento di osservazione). Inoltre un resoconto basato sulla ricostruzione di un trattamento fondato sulle impressioni del terapeuta lascia al ricercatore pochi margini di "operazionalizzabilità"; ciò accade perché la ricostruzione mnemonica degli avvenimenti è soggetta a distorsione e dunque può essere ritenuto uno strumento poco obiettivo e affidabile. Riprendendo e modificando alcune categorie proposte da Widlocher (Widlocher, 1994), abbiamo distinto le seguenti modalità di resocontazione del caso clinico. d1) Trascritto: ci riferiamo a una modalità automatizzata di registrazione del trattamento, il trascritto può essere poi redatto secondo diverse modalità. d2) Verbatim: sono i trascritti che seguono lo standard verbatim. d3) Self-Report: riguarda le diverse modalità di registrare le impressioni del paziente o del terapeuta (ad esempio, diario, inventari di personalità, questionari, etc). d4) Vignetta: riguarda gli estratti da una seduta ritenuti emblematici, gli stralci significativi del trattamento. d5) Monografia: riguarda il riassunto dell'intero trattamento. d6) No: questa voce riguarda l'assenza di trascritti.

Con l'area definita Strategie di ricerca identifichiamo le dimensioni legate ad aspetti di natura metodologica e concettuale che sono dominio del ricercatore. In quest'area ricadono le problematiche dell'unità di analisi e i problemi di ordine metodologico. Le variabili considerate sono:

e) Campione. Con questa variabile ci riferiamo al campione utilizzato nella ricerca. Sono state create le seguenti fasce: e1) Fascia 1 che equivale a $\mathrm{N}=1$. e2) Fascia 2 che comprende $\mathrm{i}$ campioni da 2 a 20 soggetti. e3) Fascia 3 da 21 a 50 soggetti. e4) Fascia 4 da 51 a 100. e5) Fascia $5 \mathrm{~N}$ > 100. e6) Esempio Illustrativo: non c'è campione, i soggetti hanno funzione descrittiva.

f) Disegno. Abbiamo adottato una tripartizione dei disegni distinguendoli in disegni che confrontano gruppi, disegni single case e studi di caso. f1) Gruppo: studi che utilizzano il confronto tra gruppi (ad esempio, le medie dei punteggi tra due gruppi). f2) Single Case studi sistematici basati sulla singola unità campionaria. f5) Studio di Caso: studi che non impiegano procedure statistiche per analizzare il caso.

g) Fonte Riguarda lo strumento di rilevazione del dato [coincide con l'observational channel di Bordin, (1974)]. Con questa variabile ci siamo chiesti: “attraverso quale modalità viene registrato il dato?". Le fonti di 
Ricerca in Psicoterapia / Research in Psychotherapy 2010; 1(13): 92-119. http://www.researchinpsychotherapy.net

rilevazione del dato sono: g1) Audiotape: il dato viene audio registrato. g2) Videotape: il dato è videoregistrato. g3) Dati Testuali: il dato viene trascritto o riassunto. g4) Test e Questionari: il dato viene rilevato attraverso l'uso di strumenti quali test o questionari. g5) Colloquio: non vi è altra registrazione del trattamento che il colloquio stesso. g6) Mista: riguarda la possibile commistione di media (audio e video, intervista e test etc.) nel registrare il dato.

h) Analisi della fonte del dato. Riguarda i criteri interpretativi utilizzati. h1) Coding: c'è una specifica unità d'analisi con un codice condiviso e sistematico che funge da dizionario nell'etichettamento e nella siglatura del documento. h2) Descrizione Aneddotica: non c'è una specifica unità d'analisi, il documento è soggetto a un' operazione ermeneutica. Sul modello dei primi casi clinici è una descrizione non sistematica degli avvenimenti affidata alle impressioni dell'autore. h3) Frequenze: modalità di analisi basata sul conteggio della frequenza di comparsa di alcuni dati (siano esse parole, frasi, etc. in termini di ricorrenze, cooccorrenze, etc.). h4) Punteggio: attribuzione di un valore numerico alle risposte date a un test o a un questionario.

i) Tipologia di Ricerca. Abbiamo suddiviso i lavori in due principali tipologie: i1) Process. i2) Process-Outcome.

j) Infine, è stata considerata la tipologia di analisi statistiche, suddivise in base alla seguente classificazione: j1) Descrittive: questa modalità comprende le medie, le frequenze, i test sul modello del $c h i$ e le misure di valutazione (che a loro volta sono costituite da tutte le misure di fedeltà, affidabilità e inter-rater). j2) Descrittivo/Inferenziali: in questa categoria ricadono gli studi che presentano sia analisi descrittive sia inferenziali (ANOVA, analisi della regressione, etc.). j3) Esplorative: questa categoria aggrega tutti gli studi che impiegano analisi fattoriali, cluster analysis, analisi delle sequenze, etc. j4) Esplorativo/Inferenziali: questa categoria comprende studi basati su analisi sia fattoriale, dei cluster, analisi della varianza, regressione, etc. j5) Inferenziale: qui troviamo studi che presentano analisi della regressione, varianza, correlazione, equazioni strutturali, e tutte le analisi basate sulla verifica delle ipotesi. j6) Mixed: in questa categoria si trovano raggruppate tutte e tre le modalità contestualmente, cioè Descrittiva, Esplorativa e Inferenziale. ji) No: assenza di statistiche.

\subsection{Campione}

La nostra letteratura di riferimento è costituita dalle riviste indicizzate che accolgono articoli riguardanti la ricerca di processo in psicoterapia. Attraverso il Database di PsycInfo (in data 7 novembre 2008 conteneva 2437 riviste) abbiamo individuato le riviste della nostra popolazione prendendo in considerazione uno span temporale di 10 anni (1998-200\%). Per essere selezionate le riviste dovevano contenere nel titolo la parola "Psychotherapy" o "Clinical Psychology". Queste due chiavi hanno generato la nostra popolazione, costituita da 40 risultati, di cui 29 corrispondenti alla chiave "Psychotherapy" e 11 alla chiave "Clinical Psychology", con una 
Ricerca in Psicoterapia / Research in Psychotherapy 2010; 1(13): 92-119. http://www.researchinpsychotherapy.net

sovrapposizione delle due chiavi nella rivista Clinical Psychology and Psychotherapy. ${ }^{3}$ Le riviste costituenti la nostra popolazione sono $39 .^{4}$

Al fine di individuare un campione rappresentativo di tale popolazione abbiamo estratto in modo randomizzato, dal decennio 1998-200\%, tre anni (2000, 2003, 2007). Per ciascuno dei tre anni abbiamo conteggiato il numero di articoli di processo contenuti in ciascuna rivista, stilandone una classifica. Abbiamo preso in considerazione le prime 13 riviste poiché su un totale di 378 articoli di processo queste ne contenevano un terzo (27\% articoli, pari all'85\%). Il nostro campione finale comprendeva così tredici riviste sulle quali abbiamo conteggiato gli articoli di processo per l'intero decennio 1998-200\%.

Complessivamente le pubblicazioni del nostro campione ammontavano a un totale di 5548 articoli. L’individuazione degli studi è avvenuta sulla base della lettura degli abstract utilizzando due criteri. Sono stati inclusi:

1. Gli articoli che trattavano aspetti teorici della ricerca di processo (ad esempio, studi concettuali, teorici).

2. Gli articoli che producevano dati sui fenomeni che osservavano (ad esempio, ricerche empiriche sia di processo puro sia di processoutcome).

\subsection{Procedura}

La selezione degli articoli è avvenuta in due fasi. Nella prima abbiamo distinto gli articoli che si occupano di processo (indipendentemente dal loro oggetto di studio/analisi) da quelli che non sono articoli di processo. Nella seconda abbiamo distinto gli articoli di processo empirici da quelli teorici.

a) Primo screening (criteri di inclusione e di esclusione). Per essere incluso in questo primo livello di screening, un articolo doveva occuparsi di:

- analizzare/studiare il processo terapentico modellizandolo empiricamente oppure quale oggetto di riflessione teorica. Abbiamo così inserito articoli di processo che trattavano aspetti concettuali, empirici o entrambi.

Sono stati inclusi gli studi qualitativi, gli studi di metodo e quelli di trattamento, che presentavano l'impiego di uno strumento, purché questi misurasse un aspetto del processo; sono state inoltre incluse le meta-analisi, le reviews e i commenti agli articoli. Sono stati esclusi gli editoriali, i book reviews, i necrologi, la biografia di autori, le corrispondenze, le note editoriali.

In questa prima fase, dall'insieme dei 5548 articoli abbiamo estratto 557 articoli di processo (sia processo che process outcome) complessivi (10.4\% del totale) e 4491 articoli non di processo ( $89.96 \%$ del totale).

\footnotetext{
${ }^{3}$ D'ora in poi utilizzeremo gli acronimi delle riviste rimandando all'Appendice A per i titoli estesi.

${ }^{4}$ Per l'elenco delle riviste che costituiscono la nostra popolazione si veda l'Appendice A.
} 
Ricerca in Psicoterapia / Research in Psychotherapy 2010; 1(13): 92-119. http://www.researchinpsychotherapy.net

b) Secondo screening (la cui selezione è avvenuta attraverso la rilettura degli abstract) è stato condotto per differenziare due tipologie di articoli che abbiamo così definito:

- articoli di Processo (per una definizione di processo si veda il paragrafo metodo) di $1^{\circ}$ Livello, cioè articoli che producono dati sul fenomeno in osservazione o che utilizzano dati da precedenti ricerche per produrre ulteriori dati. Per poter essere considerato di $1^{\circ}$ livello uno studio doveva analizzare variabili di processo misurate empiricamente oppure doveva presentare una relazione rilevata empiricamente (positiva negativa o nulla) tra variabili di processo e variabili di esito in psicoterapia;

- articoli di Processo di $2^{\circ}$ Livello (articoli teorici concettuali, metaanalisi, review).

Gli articoli di Processo di primo livello sono articoli empirici che producono dati sul fenomeno in osservazione, siano essi resoconti clinici o misurazioni sistematiche. Gli articoli di Processo di secondo livello sono articoli teorici, meta-analisi, articoli di risposta ad altri studi, oppure review.

Dei 557 articoli di processo $310(56 \%)$ sono stati inclusi tra gli articoli di primo livello, mentre i restanti 247 (44\%) sono stati inclusi tra gli articoli di processo di secondo livello. Le nostre analisi (descrittive e analisi delle corrispondenze multiple) si sono concentrate sui $\mathbf{3 1 0}$ articoli di primo livello.

Gli articoli di processo nel campione di riviste da noi considerato occupano un margine ancora esiguo (10\%) esiguo (Tabella 1). Inoltre il rapporto tra articoli di processo di $1^{\circ}$ e di $2^{\circ}$ livello appare lievemente sbilanciato a favore del primo ( $56 \%$ vs $44 \%$ ) il campo della ricerca appare pertanto suddiviso in modo paritario tra ricerca empirica e riflessione teorica. Con un maggiore livello di dettaglio possiamo osservare il rapporto esistente (per ciascuna rivista) tra articoli di processo di $1^{\circ}$ e di $2^{\circ}$ livello (Figura 1) e la distribuzione percentuale degli articoli di processo di $1^{\circ}$ livello per ciascuna rivista (Tabella 2). In particolare le riviste che presentano il maggior numero di articoli di processo di $1^{\circ}$ livello e che adottano quindi un approccio prevalentemente empirico sono: PR con il $22 \%$ della distribuzione, CPR (13\%), a seguire PTRPT (12\%) ed il JCCP (12\%). 
Ricerca in Psicoterapia / Research in Psychotherapy 2010; 1(13): 92-119. http://www.researchinpsychotherapy.net

Tabella 1. Segmentazione dell'unità d'analisi.

\begin{tabular}{|c|c|c|}
\hline Unita' & Quantita' & Descrizione \\
\hline Articoli & 5548 & $\begin{array}{l}\text { Totale complessivo degli articoli } \\
\text { presenti nelle } 13 \text { riviste } \\
\text { selezionate }\end{array}$ \\
\hline Articoli di processo complessivi & 557 & $\begin{array}{l}\text { Totale complessivo degli articoli } \\
\text { di processo individuati nelle } \\
\text { riviste elencate }\end{array}$ \\
\hline Articoli di processo $1^{\circ}$ livello & 310 & $\begin{array}{l}\text { Totale articoli di processo empici } \\
\text { che presentano analisi attraverso } \\
\text { dati di prima mano }\end{array}$ \\
\hline Articoli di processo $2^{\circ}$ livello & 247 & $\begin{array}{l}\text { Totale articoli di processo che } \\
\text { comprendono articoli } \\
\text { concettuali, di metodo, di meta- } \\
\text { analisi e review }\end{array}$ \\
\hline Articoli non di processo & 4991 & Totale residuo \\
\hline
\end{tabular}

\subsection{Analisi della produttività}

Il parametro di qualità della ricerca che sarà considerato è quello della produttivitá. L'analisi della produttività è un parametro bibliometrico che analizza il numero e la tipologia di lavori che sono stati prodotti, separandoli dall'impatto che questi producono sulla comunità scientifica. In questo modo si tengono disgiunte informazioni che spesso in letteratura appaiono sovrapposte, quali ad esempio la valutazione di una pubblicazione sulla base dell'Impact Factor della rivista.

Gli strumenti di analisi della produttività che abbiamo utilizzato sono lo studio della distribuzione degli articoli per anno, e l'eterogeneità dei dati usando l'indice di Gini.

In riferimento a tutti gli articoli sia di processo sia non, pubblicati sul campione delle nostre riviste si osserva un trend complessivo prevalentemente in crescita (Figura 2). Possiamo sostenere quindi con certezza che vi è stato un notevole incremento del numero di pubblicazioni nell'ultimo decennio. In particolare a partire dall'anno 2004 .

Gli articoli di process di $1^{\circ}$ Livello presentano un trend ad $\mathrm{U}$ prodotto da un decremento iniziale, con una stabilizzazione successiva che va dal 2000 al 2004, e subiscono un repentino innalzamento a partire dal 2004, sino al 2007 (Figura 3). Gli articoli di process di 2 livello presentano un andamento a zig zag con un improvviso innalzamento dall'anno 2003, incremento costante sino al $200 \%$. 
Ricerca in Psicoterapia / Research in Psychotherapy 2010; 1(13): 92-119. http://www.researchinpsychotherapy.net

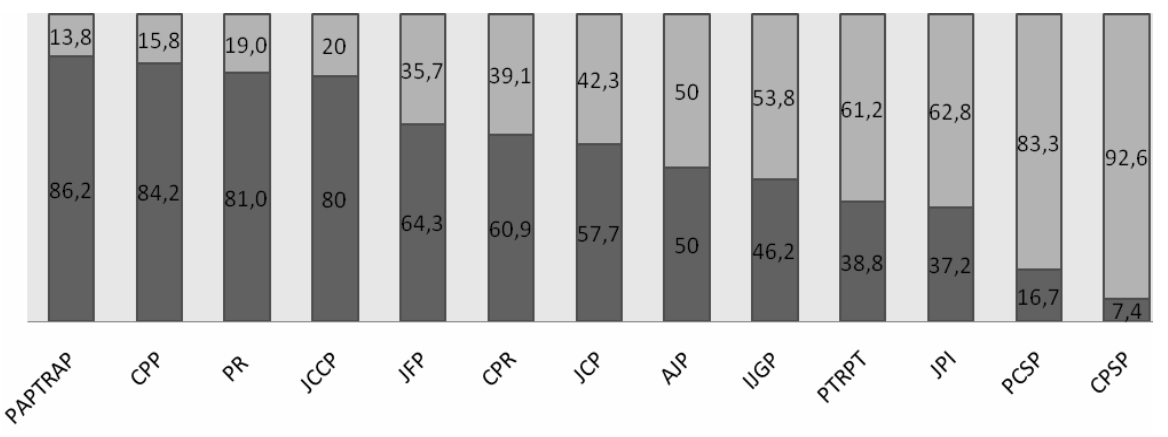

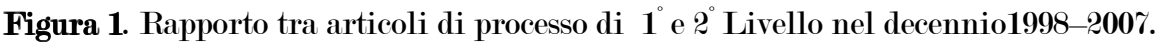

Tabella 2. Distribuzione degli articoli di $1^{\circ}$ Livello per rivista.

\begin{tabular}{lcc}
\hline \multicolumn{1}{c}{ RIVISTA } & Frequenza & \% \\
\hline Psychotherapy Research & 68 & 21,9 \\
Counselling \& Psychotherapy Research & 39 & 12,6 \\
Psychotherapy: Teory, Research, Practice and Training & 38 & 12,3 \\
Journal of Consulting and Clinical Psychology & 36 & 11,6 \\
Psychology and Psychotherapy: Theory, Research and Practice & 25 & 8,1 \\
International Journal of Group Psychotherapy & 24 & 7,7 \\
Journal of Psychotherapy Integration & 18 & 5,8 \\
Clinical Psychology and Psychotherapy & 16 & 5,2 \\
Journal of Family Psychotherapy & 16 & 5,2 \\
Journal of Clinical Psychology & 15 & 4,8 \\
American Journal of Psychotherapy & 9 & 2,9 \\
Pragmatic Case Studies in Psychotherapy & 4 & 1,3 \\
Clinical Psychology: Science and Practice & 2 & 0,6 \\
\hline
\end{tabular}


Ricerca in Psicoterapia / Research in Psychotherapy 2010; 1(13): 92-119. http://www.researchinpsychotherapy.net

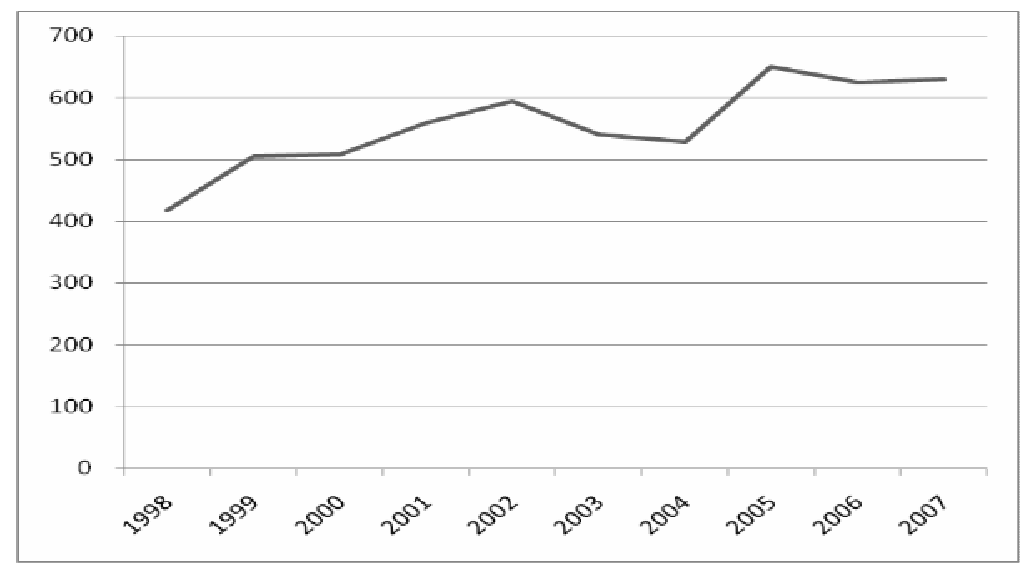

Figura 2. Trend Articoli 1998-200\%.

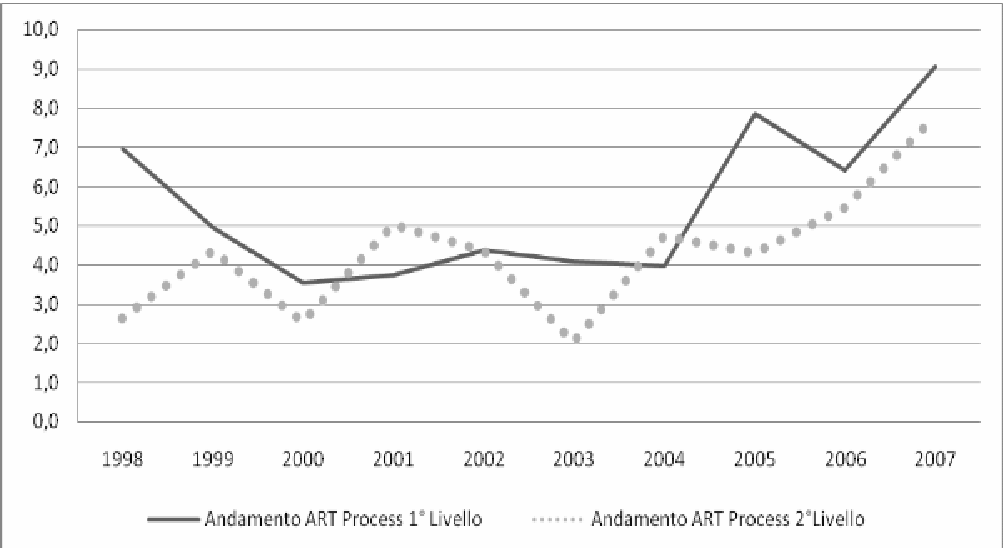

Figura 3. Confronto andamento tra gli articoli di 1 e di $2{ }^{\circ}$ Livello.

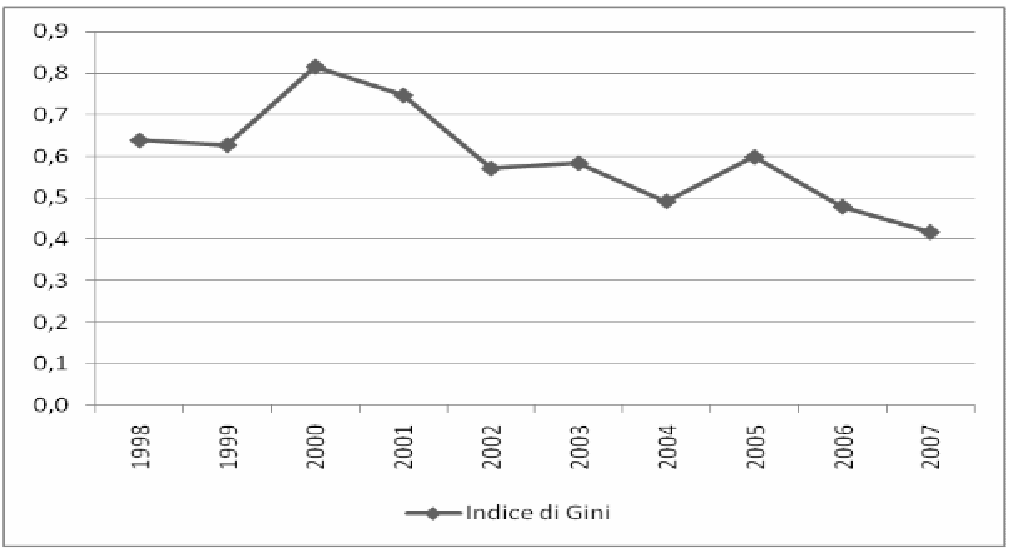

Figura 4. Indice di Gini. 
Ricerca in Psicoterapia / Research in Psychotherapy 2010; 1(13): 92-119. http://www.researchinpsychotherapy.net

i pubblicazioni che trattano di ricerca di processo tende negli anni a Si osserva inoltre un picco d'innalzamento con esordio nel 2003 negli articoli di process di 2 Livello, incremento seguito nel 2004 dagli articoli di process di 1 Livello. La correlazione ci informa che c’è una tendenza positiva tra le due variabili di poco sotto i valori di significatività (con $\alpha=0.05$ e gdl ( $\mathrm{n}-$ 2) pari a 8 , il valore critico è 0.549 , il nostro punteggio è pari a 0.537 ).

L'indice di Gini (Figura 4) è uno stimatore appropriato riguardo l'eterogeneità delle pubblicazioni prodotte nel tempo, questi è una misura di rapporto basata sulla diseguaglianza di una distribuzione. L'indice varia tra 0 e 1, dove con 0 si intende massima omogeneità e 1 è massima eterogeneità. Il grafico ci mostra come nel tempo si sia passati da una forte eterogeneità iniziale a una graduale omogeneità. In altre parole il numero d

"spalmarsi" nelle riviste in modo sempre più uniforme. Ciò costituisce indirettamente anche un indice di diffusione e permeabilità del settore di ricerca.

\subsection{Analisi descrittive}

Riguardo la distribuzione delle dimensioni della griglia si osserva che nell'Area Geografica assistiamo a uno sbilanciamento degli articoli di $1^{\circ}$ livello nell'area degli USA che da sola copre la metà della distribuzione. Il secondo polo è costituito dall'Europa con il $30 \%$. Abbastanza rappresentativo è il contributo del Canada con il $\mathbf{1 3} \%$. Le altre aree sono poco o per nulla rappresentate. La ricerca empirica sul processo è appannaggio quindi degli Stati Uniti e dell'Europa ed è scarsamente rappresentata dalle altre aree.

Riguardo l'Area Psicoterapica di intervento, l'area maggiormente rappresentata è la modalità Altro (24\%), seguono gli interventi di stampo psicodinamico PDT (21\%) e gli interventi misti (19\%).

Riguardo la Tipologia di Autore, la principale provenienza istituzionale è l'Università. Bisogna tener presente che il conteggio era effettuato sulla base dei primi due autori. Il dato quindi testimonia anche di un'elevata "endogamia", confermata dal fatto che l'indice più basso $(9 \%)$ è costituito dalla modalità entrambi, che appunto vede uniti nella stessa ricerca clinici e accademici. Il rapporto quindi ricerca-clinica, appare ancora esigno e sbilanciato prepotentemente a favore dell'Università. I clinici in qualità di ricercatori coprono invece il 13\% della distribuzione. Riguardo al Campione, si osserva una equidistribuzione della variabile nelle diverse fasce. La più ampia è costituita dalla fascia 2-20.

Il principale Disegno di Ricerca è il Group Design che occupa la metà dell'insieme $(50 \%)$. Seguono i Disegni Single Case con il $26 \%$. Infine lo studio di caso (24\%). Prevale quindi la ricerca sistematica. Un quarto del campione invece predilige un approccio più descrittivo.

Relativamente alla Fonte del dato, lo strumento d'elezione è costituito dall'uso dei Test e Questionari (38\%). Segue l'audiotape (21\%) che, se sommato con il videotape (12\%), costituisce complessivamente il $33 \%$ del totale, poi i dati testuali (19\%). Il colloquio occupa una piccola porzione (8\%) dell'insieme, mentre le tecniche miste (ad esempio, andio e videotape insieme, oppure test e videotape) risultano le meno rappresentative ( $2 \%)$.

La principale modalità di Analisi della Fonte del Dato è costituita dall'assegnazione di un punteggio (40\%), seguita dalla codifica (34\%) e dalla 
104

Ricerca in Psicoterapia / Research in Psychotherapy 2010; 1(13): 92-119. http://www.researchinpsychotherapy.net

descrizione aneddotica (21\%) il valore più basso è costituito dalle Frequenze $(5 \%)$.

Riguardo la Tipologia di resoconto, nei nostri articoli prevale l'assenza di Resoconto (50\%). Segue il resoconto basato sul Trascritto $(26 \%)$ che sommato con la modalità Verbatim (12\%) raggiunge il 38\% del totale. Il restante $12 \%$ è suddiviso equamente tra le modalità Self-Report, Vignetta e Monografia.

Riguardo la Tipologia di analisi statistiche, una prima differenza riguarda l'impiego o meno di procedure statistiche, dato che contrappone da un lato il $66 \%$ e dall'altro il 34\% (modalità NO). Ciò significa che più di un terzo degli studi non adotta procedure statistiche. Pertanto buona parte della ricerca di process (105 studi su 310) si caratterizza a tutt'oggi grazie a un approccio più descrittivo e aneddotico. La tipologia statistica invece più rappresentativa è quella inferenziale (34\%). Se abbinate alle descrittive inferenziali $(16 \%)$ raggiungiamo la metà del campione. Le indagini esplorative occupano il $6 \%$, mentre quelle che abbinano l'esplorazione all'inferenza costituiscono il 5\%. La porzione più piccola è data dai metodi Mixed.

Riguardo infine alla Tipologia di Ricerca, la ricerca di processo nei nostri studi si suddivide in modo egualitario tra processo e process-outcome con un lievissimo sbilanciamento per le ricerche di processo (Tabella 3).

\subsection{Analisi inferenziali}

È stata condotta un'Analisi delle corrispondenze Multiple ${ }^{5}$ sulle variabili della nostra griglia con l'obiettivo di mappare i diversi modelli di processo presenti e di analizzare dimensioni sintetiche in grado di spiegarne $i$ reciproci posizionamenti. Dalla nostra analisi abbiamo estratto i primi due fattori che spiegano il $48 \%$ dell'inerzia.

1 Fattore: Modello d'analisi. Il primo fattore (Tabella 4) spiega il $32 \%$ dell'inerzia; questa rappresenta e sintetizza due differenti modelli di analisi utilizzati nella ricerca di processo in psicoterapia: un modello EMPIRICO e un modello CLINICO (in appendice B si riportano gli autovalori e l’inerzia ricalcolata con la formula di Benzecri). Il semiasse negativo è saturato dalle modalità che comprendono l'uso dei TEST E DEI QUESTIONARI; la ricerca è di PROCESS OUTCOME; si predilige il confronto tra gruppi (GROUP DESIGN); si utilizzano prevalentemente campioni molto ampi (maggiori di cento soggetti o comunque tra i cinquanta e i cento); le analisi statistiche adottano prevalentemente modelli INFERENZIALI di verifica delle ipotesi; la funzione della resocontazione è assente. Prevalgono le terapie MULTIPLE, (cioè quelle di confronto). Le riviste espressioni di questo semiasse sono in primis il JCCP (V. TEST - 6.42), seguono, anche se con un valore meno significativo, PR (V. TEST - 2.99) e JCP (V. TEST 2.65).

\footnotetext{
${ }^{5}$ Per tutti gli aspetti tecnici relativi all' ACM e alle procedure adottate si rimanda all'appendice B.
} 
105

Ricerca in Psicoterapia / Research in Psychotherapy 2010; 1(13): 92-119. http://www.researchinpsychotherapy.net

Tabella 3. Descrizione delle variabili della griglia in valori assoluti e percentuali ACM.

\begin{tabular}{|c|c|c|}
\hline VARIABILE & Frequenza & $\%$ \\
\hline \multicolumn{3}{|l|}{ Area Geografica } \\
\hline USA & 154 & 49,7 \\
\hline Europa & 93 & 30,0 \\
\hline Canada & 41 & 13,2 \\
\hline Australia & 9 & 2,9 \\
\hline Asia & 7 & 2,3 \\
\hline Sud America & 5 & 1,6 \\
\hline Africa & 1 & 0,3 \\
\hline \multicolumn{3}{|c|}{ Area Psicoterapica di Intervento } \\
\hline Altro & 76 & 24,5 \\
\hline PDT & 65 & 21,0 \\
\hline Miste & 58 & 18,7 \\
\hline Multiple & 42 & 13,5 \\
\hline Ermeneutica & 30 & 9,7 \\
\hline Cognitiva & 21 & 6,8 \\
\hline Non specificata & 18 & 5,8 \\
\hline \multicolumn{3}{|l|}{ Tipologia Autore } \\
\hline Accademico & 242 & 78,1 \\
\hline Clinico & 40 & 12,9 \\
\hline Entrambi & 28 & 9,0 \\
\hline \multicolumn{3}{|l|}{ Campione } \\
\hline Fascia $2-20$ & 65 & 21,0 \\
\hline Fascia $>100$ & 53 & 17,1 \\
\hline Fascia 21-50 & 52 & 16,8 \\
\hline Esempio illustrativo & 52 & 16,8 \\
\hline Fascia 1 & 49 & 15,8 \\
\hline Fascia 51-100 & 39 & 12,6 \\
\hline \multicolumn{3}{|l|}{ Disegno } \\
\hline Group design & 155 & 50 \\
\hline Single case & 80 & 25,8 \\
\hline Studio di caso & 75 & 24,2 \\
\hline \multicolumn{3}{|l|}{ Fonte } \\
\hline Test e questionari & 118 & 38,1 \\
\hline Audiotape & 66 & 21,3 \\
\hline Dati testuali & 58 & 18,4 \\
\hline Videotape & 38 & 12,3 \\
\hline Colloquio & 24 & 7,7 \\
\hline Mista & $\gamma$ & 2,3 \\
\hline \multicolumn{3}{|c|}{ Analisi della Fonte dei Dati } \\
\hline Punteggio & 125 & 40,3 \\
\hline Coding & $10 \%$ & 34,5 \\
\hline Descrizione aneddotica & 64 & 20,6 \\
\hline Frequenze & 14 & 4,5 \\
\hline \multicolumn{3}{|l|}{ Resoconto } \\
\hline No & 154 & 49,7 \\
\hline Trascritto & 81 & 26,1 \\
\hline Verbatim & 37 & 11,9 \\
\hline Monografia & 14 & 4,5 \\
\hline Self report & 12 & 3,9 \\
\hline Vignette & 12 & 3,9 \\
\hline \multicolumn{3}{|c|}{ Tipologia di Analisi Statistiche } \\
\hline No & 105 & 33,9 \\
\hline Inferenziale & 75 & 24,2 \\
\hline Descrittivo/inferenziali & 49 & 15,8 \\
\hline Descrittive & 33 & 10,6 \\
\hline Esplorative & 20 & 6,5 \\
\hline Esplorativo/inferenziale & 16 & 5,2 \\
\hline Mixed & 12 & 3,9 \\
\hline \multicolumn{3}{|l|}{ Tipologia di Ricerca } \\
\hline Process & 158 & 51,0 \\
\hline Process Outcome & 152 & 49,0 \\
\hline
\end{tabular}


Ricerca in Psicoterapia / Research in Psychotherapy 2010; 1(13): 92-119. http://www.researchinpsychotherapy.net

Il semiasse positivo è saturato invece in primis dalla variabile Tipologie di Analisi Statistiche, che presenta la modalità NO; segue la variabile Disegno che predilige lo STUDIO DI CASO; a seguire la variabile Analisi della fonte del dato con la modalità DESCRIZIONE ANEDDOTICA degli eventi; assenza di Campione abbiamo solo ESEMPI ILLUSTRATIVI; la fonte dei dati fa affidamento sul COLLOQUIO; si predilige l'uso delle VIGNETTA nella presentazione del caso; prevale l'approccio ERMENEUTICO; la ricerca è prevalentemente di PROCESS. Questo semiasse racchiude e descrive un approccio in cui prevale l'interpretazione clinica del fenomeno oggetto di analisi. Le riviste maggiormente rappresentative sono il JFP (V. TEST 4.68), il CPR (V. TES'T 3.83) e il JPI $(3.75)$.

Emergono due modi incommensurabili di intendere la ricerca di processo, che riflettono a loro volta una differenza nelle premesse epistemologiche e concettuali alla base del concetto di significatività: è una misura statistico-probabilistica per i primi, è un indice della "pregnanza" della relazione clinica per $\mathrm{i}$ secondi.

$2^{\circ}$ Fattore: Metodo interpretativo. Questo secondo fattore (Tabella 4) spiega il $16 \%$ dell’inerzia e insieme con il primo il $48 \%$ dell'inerzia totale. Il secondo fattore può essere considerato come una specificazione, una risposta al primo fattore. Questi - attraverso le categorie con le quali interroga i dati, e attraverso l'unità di analisi prescelta - descrive un continuum lungo il quale si dispongono differenti tipologie di disegni: single case, studi qualitativi, studi di caso.

Sul semiasse negativo INTERPRETAZIONE SISTEMATICA, i principali contributi provengono dalla modalità CODING, AUDIOTAPE, SINGLE CASE, seguita dal TRASCRITTO. L'Area Geografica è rappresentata dall'EUROPA. Le riviste che riflettono il semiasse sono il CPR (V. TEST - 5.08) e PR (V. TEST - 4.58).

Sul semiasse positivo che è stato definito INTERPRETAZIONE NON SISTEMATICA la modalità che satura principalmente è la DESCRIZIONE ANEDDOTICA, seguita dallo STUDIO DI CASO e dal COLLOQUIO; non vi è indagine campionaria, bensì soggetti utilizzati unicamente con lo scopo di presentare la procedura o il modello. Le principali riviste sono IJGP (V. TEST 5.5\%) e il JPI (V. TEST 3.96).

Il fattore riflette due differenti metodi interpretativi: il primo è caratterizzato da rigore delle procedure, garantito dalla codifica sistematica e puntuale della fonte del dato che intensifica sia la validità di costrutto, sia la possibilità di verifica del processo di operazionalizzazione, poiché identifica una fonte specifica e costante per analizzare il dato. Di contro il metodo NON SISTEMATICO si basa sull'inferenza del terapeuta e sulla sua personale ricostruzione degli eventi,; il resoconto redatto tramite i colloqui appare la fonte esaustiva e auto-evidente dei risultati raggiunti, il che rende l'operatore (psicologo /ricercatore/ terapeuta) l'unico strumento di verifica. 
Ricerca in Psicoterapia / Research in Psychotherapy 2010; 1(13): 92-119. http://www.researchinpsychotherapy.net

Tabella 4. Valori Test dell'ACM.

\section{$1{ }^{\circ}$ FATTORE}

\begin{tabular}{|c|c|c|}
\hline \multicolumn{3}{|l|}{ EMPIRICO } \\
\hline VARIABILE & MODALITA' & V. TEST \\
\hline Fonte & Test e questionari & $-12,59$ \\
\hline Analisi della fonte dei dati & Punteggio & $-12,31$ \\
\hline Tipologia di ricerca & Process out come & $-11, \gamma$ \\
\hline Disegno & Group design & -10 \\
\hline Campione & Fascia > 101 & $-8,29$ \\
\hline Tipologia di analisi statistiche & Inferenziale & $-7,64$ \\
\hline Resoconto & No & $-7,26$ \\
\hline Campione & Fascia 51-100 & $-5,02$ \\
\hline Tipologia di analisi statistiche & Descrittivo/inferenzenziale & $-4,87$ \\
\hline Area psicoterapica & Multiple & $-4,8$ \\
\hline \multicolumn{3}{|l|}{ CLINICO } \\
\hline Tipologia di analisi statistiche & No & 15,43 \\
\hline Disegno & Studio di caso & 13,74 \\
\hline Analisi della fonte dei dati & Descrizione aneddoti & 13,44 \\
\hline Tipologia di ricerca & Process & 12,76 \\
\hline Campione & Esempio illustrativo & 12,05 \\
\hline Fonte & Colloquio & 8,87 \\
\hline $\begin{array}{l}\text { Fonte } \\
\text { Resoconto }\end{array}$ & $\begin{array}{l}\text { Dati testuali } \\
\text { Vignette }\end{array}$ & $\begin{array}{l}7,38 \\
6\end{array}$ \\
\hline Resoconto & Monografia & 5,87 \\
\hline Area psicoterapica & Ermeneutica & 5,75 \\
\hline \multicolumn{3}{|c|}{$2^{\circ}$ FATTORE } \\
\hline VARIABILE & MODALITA' & V. TEST \\
\hline \multicolumn{3}{|c|}{ INTERPRETAZIONE SISTEMATICA } \\
\hline Analisi della fonte dei dati & Coding & $-11,33$ \\
\hline Fonte & Audiotape & $-9,97$ \\
\hline Disegno & Single case & $-8,75$ \\
\hline Resoconto & Trascritto & $-8,19$ \\
\hline Campione & Fascia 1 & $-7,83$ \\
\hline Resoconto & Verbatim & $-7,46$ \\
\hline \multicolumn{3}{|c|}{ INTERPRETAZIONE NON SISTEMATICA } \\
\hline Analisi della fonte dei dati & Descrizione aneddotica & 9,98 \\
\hline Disegno & Studio di caso & 9,28 \\
\hline Fonte & Colloquio & 9,22 \\
\hline Campione & Esempio illustrativo & 9,22 \\
\hline Resoconto & No & 9,09 \\
\hline
\end{tabular}


Ricerca in Psicoterapia / Research in Psychotherapy 2010; 1(13): 92-119. http://www.researchinpsychotherapy.net

\section{Discussione}

Nell'arco temporale 1998-200\% si riscontra un complessivo aumento del numero di pubblicazioni nelle riviste (a prescindere dagli specifici settori di ricerca: process/non process). Malgrado ciò, è ancora esiguo il numero complessivo di articoli sulla ricerca di processo (10\% sul totale).

In compenso si registrano una permeabilità crescente del settore di ricerca e una sua capillarizzazione nelle riviste.

Riguardo la distribuzione delle nostre variabili si osserva che è il paziente il focus principale degli studi sul processo, mentre lo strumento principale di rilevazione del dato è costituito dal questionario. Riguardo alle tecniche di analisi statistica prevalgono le tipologie di natura inferenziale, dove la ricerca è finalizzata alla verifica di un'ipotesi. Occupano un considerevole spazio anche studi che non utilizzano procedure statistiche.

I dati nel loro complesso presentano un'elevata eterogeneità di strumenti (344 su 310 articoli) di metodi, di disegni. La mole considerevole di studi condotti senza l'impiego di tecniche statistiche, e senza l'uso di unità campionarie è il segnale della presenza simultanea di visioni incommensurabili nella ricerca.

Riguardo il rapporto clinica e ricerca rimane ampio il gap testimoniato dalla quasi totale assenza di ricerche che coinvolgono insieme clinici e i ricercatori a conferma della condizione di scissione riscontrato nei precedenti studi.

Sono emersi due principali modelli epistemologici: il modello d'analisi e quello interpretativo. Questi organizzano tre principali approcei alla ricerca sul processo che abbiamo definito: Congetturale, Sperimentale e Misto. Questi differenti approcei sollevano interrogativi riguardo al concetto di significatività, alla scelta delle unità d’analisi e al rapporto tra oggetto della ricerca e metodologia adottata.

L'approccio Congetturale si caratterizza per la prevalenza di studi di taglio concettuale, non utilizza strumentazione statistica né disegni di ricerca, né una specifica e univoca unità d’analisi; diversi lavori cercano la propria validazione mediante la presentazione dei casi clinici stessi (ad esempio, presentando come evidenza delle proprie asserzioni, i resoconti delle sedute). In alcuni di questi studi il processo sembra essere un pretesto, diviene il luogo di applicazione della tecnica d'intervento, finisce pertanto sullo sfondo e viene a costituire una cornice inerte, non informativa. Questo approccio riflette prevalentemente l'area geografica relativa all'Asia.

L'approccio Sperimentale valuta l'efficacia clinica sulla base della significatività probabilistica calcolata attraverso misure (generalmente le medie dei gruppi) della variabilità between group. Alcuni articoli che ricadono in questo modello indagano su specifiche patologie sostenendo un modello d'intervento d'elezione. Questo approccio è espressione dell'area geografica relativa agli USA. 
Ricerca in Psicoterapia / Research in Psychotherapy 2010; 1(13): 92-119. http://www.researchinpsychotherapy.net

Con l'approccio Misto s'intende prevalentemente la ricerca single case. Attraverso questo approccio l'obiettivo è quello di conciliare la contingenza e unicità del trattamento con misure in grado di poter generalizzare i risultati ottenuti. Questi riflette prevalentemente l'area geografica relativa all'Europa.

La Process Research attraverso l'analisi di questi studi si presenta come un settore di ricerca eterogeneo. Essa si organizza in prima istanza sui modelli di analisi e sulle metodologie interpretative attraverso i quali osserva i propri oggetti. Ciò significa che il modello d’analisi (nelle sue declinazioni, empirico e clinico) e la strategia interpretativa (sistematica e non sistematica) fungono da organizzatori, diremmo da "interpretanti" della realtà fenomenica. Sono queste le principali biforcazioni dalle quali originano differenti approcei. Il modello d'analisi e la metodologia interpretativa sono a loro volta due modelli che si definiscono dialetticamente in ragione del paradigma epistemologico di riferimento. Quando la ricerca interpreta la Psicologia in quanto scienza nomotetica, essa è prevalentemente finalizzata all'individuazione di leggi invarianti dove la clinica svolge una funzione applicativa, coincide in altre parole con l'esercizio di una prassi standardizzata costruita sui risultati prodotti dalla prima. Viceversa, quando la ricerca interpreta la Psicologia quale scienza del particolare, si avvale prevalentemente dell'unicità dell'esperienza clinica quale strumento conoscitivo d'elezione ipotizzando una contingenza scevra da ogni possibilità di generalizzazione e verifica. Se da un lato quindi si rifiuta una scienza scissa dalla prassi, dall'altro si rifiutano modelli i cui criteri di verifica siano mero appannaggio delle descrizioni cliniche. Una sintesi tra le dicotomie precedenti è rintracciabile nell'approccio Misto che si realizza prevalentemente nella ricerca single case. Questi appare, sia nella sua proposta concettuale che metodologica, un modo di conciliare la contingenza e unicità del trattamento con misure e procedure in grado di generalizzare $i$ risultati ottenuti. Ciò grazie anche ai progressi relativi alla metodologia idiografica e al suo sviluppo finalizzato a individuare strategie in grado di favorire la generalizzazione dei risultati tra $i$ casi [si veda al riguardo Moleenar \& Valsiner (2005)].

\subsection{Limiti e sviluppi}

Lo studio si è limitato alle pubblicazioni scritte in lingua inglese. È possibile ampliare il numero di riviste e analizzare gli studi di processo di $2^{\circ}$ livello. L'obiettivo delle prossime ricerche riguarderà: a) l'ampliamento dello span temporale e del numero delle riviste; saranno inoltre inserite altre variabili: scopo, obiettivi, strumenti, costrutto, vertice d'analisi, unità di osservazione, unità d'analisi, aspetto del processo e fase temporale; b) l'analisi dei profili delle riviste, che potrebbero rivelarsi utili indicatori di una possibile "mappa concettuale" della ricerca sul processo. 
Ricerca in Psicoterapia / Research in Psychotherapy 2010; 1(13): 92-119. http://www.researchinpsychotherapy.net

\section{Bibliografia}

American Psychological Association. (2005). Report of the 2005 Presidential Task Force on evidence-based practice. Washington, DC: Author.

Armati, M.C., Caricati, D., Langher, V., Portaleone, A., \& Zoppi L. (1994). Rapporto tra processo e risultato nella ricerca in psicoterapia. Polarita, 8 (3), 505-512.

Aveline, M., \& Shapiro, D. (1995). Research Foundations for Psychotherapy Practice. Chichester, England: Wiley.

Beck, A.T., Rush, A.J., Shaw, B.F., \& Emery G. (1979). Cognitive Therapy of Depression. New York: The Guilford Press.

Bergin, A., \& Garfield, S. (2004). Handbook of Psychotherapy and Behavior Change: An empirical Analysis. New York: Wiley.

Bergin, A.E., \& Strupp, H.H. (1972). Changing Frontiers in the Science of Psychotherapy. Chicago: Aldine-Atherton.

Beutler, L.E., \& Crago, M. ( 1991). Psychotherapy research: an international review of programmatic studies. Washington, DC: American Psychological Association.

Beutler, L.E., Machado, P.P.P., \& Neufeldt, S.A. (1994). Therapist variables. In A.E. Bergin \& S.L. Garfield (Eds.), Handbok of Psychotherapy and Behavior Change (4th Ed.) (pp. 229-269). New York: Wiley.

Beutler, L.E., \& Clarkin, J.F. (1990). Systematic Treatment Selection. New York: Brunner/Mazel.

Beutler, L.E., Kim, E.J., Davison, E., Karno, M., \& Fisher, D. (1996). Research contributions to improving managed health care outcomes. Psychotherapy, 33, 197-206.

Bongar, B., \& Beutler, L.E., (1994). Foundations of Psychotherapy: Theory, Research, and Practice. New York: Oxford University Press.

Bordin, E.S. (1975, June). The Working alliance: basis for a general theory of psychotherapy. Paper presented at the 6th annual meeting of the Society for Psychotherapy Research,Boston, MA.

Bordin, E.S. (1974). Research Strategies in psychotherapy. New York: Wiley Interscience.

Borkenhagen, A., Decker, O., Brahler, E., \& Strauss, B. (2002). Bibliometric analysis - The Journal of the Society for Psychotherapy Research and the diversity of Psychotherapy Research: a compilation and comparison of North American and European contributions. Psychotherapy Research, 12(4), 491-514.

Campbell, L.F. (1996). The treatment outcome pursuit: a mandate for the clinical and researcher working alliance. Psychotherapy, 33, 190-96.

Carcione, A., Conti, L., \& Semerari, A. (2007). Le ricerche in psicoterapia con la scala di valutazione della metacognizione (SWAM): Applicazioni e prospettive. In G. Nicolò \& S. Salvatore (Eds.), La ricerca sui risultati e sul processo in psicoterapia (pp.320-335) Roma: Carlo Amore.

Castonguay, L.G., \& Beutler, L.E. (2006). Principles of Therapentic Change that Work. New York: Oxford University Press.

Caviglia, G., \& Salvatore, S. (1993). Un modello metodologico di verifica dell'efficacia delle psicoterapie come validazione scientifica della teoria 
Ricerca in Psicoterapia / Research in Psychotherapy 2010; 1(13): 92-119. http://www.researchinpsychotherapy.net

della tecnica. In A. Zabonati, P. Migone \& G. Maschietto (Eds.), La validazione scientifica delle psicoterapie psicoanalitiche . Mestre, VE: IPAR.

Claghorn, J.L. (1976). Successful Psychotherapy. New York: Brunner/Mazel.

Cook, T.D., \& Campbell, D.T. (1979). Quasi-experimentation: Design \& Analysis Issues for Field Settings. Boston: Houghton.

Dazzi, N., Lingiardi, V., \& Colli, A. (2006). La ricerca in psicoterapia. Milano: Cortina.

De Robbio, A. (2007). Analisi citazionale e indicatori bibliometrici nel modello Open Access. Bollettino AIB, 47(3), 257-28\%.

Di Loreto, A.O. (1971). Comparative Psychotherapy: An Experimental Analysis. Chicago: Aldine-Atherton.

Elliott, R. (1989). Comprehensive process analysis: understanding the change process in significant therapy events. In M.J. Packer \& R. B. Addison (Eds.), Entering the Circle: Ermenentic Investigation in Psychology (pp.165-184). Albany: State University of New York Press.

Elliott, R. (1991). Five dimensions of therapy process. Psychotherapy Research, 1, 92-103.

Fava, E., (200\%). Gli strumenti di analisi. In G. Nicolò \& S. Salvatore (Eds.), La ricerca sui risultati e sul processo in psicoterapia (pp. 181186). Roma: Carlo Amore.

Fensterheim, H., \& Raw, SD. (1996). Psychotherapy research is not psychotherapy practice. Clinical Psychological Science Practices. 4, 16881.

Fonagy, P., \& Moran, G. (1993). Single case Research. In N. Miller \& L. Luborsky (Eds.), Handbook of Psychodynamic Treatment Research (pp. 62-95). New York: Basic Books.

Frank, J.D., Hoehn-Saric, R., Imber, S.D., Liberman, B.L., \& Stone, A.R. (1978). Effective Ingredients of Successful Psychotherapy. New York: Brunner/Mazel.

Garfield, E. (2006). The History and Meaning of the Journal Impact Factor. $J A M A, 4,295-301$.

Garfield, S. L. (1990). Issues and Methods in Psychotherapy Process Research. Journal of Consulting and Clinical Psychology, 58(3), 273280.

Goldfried, M.R., Greenberg, L.S., \& Marmar, C. (1990). Individual psychotherapy: process and outcome. Annual Review of Psychology, 41, $659-88$.

Goldfried, M.R., \& Wolfe, B.E. (1996). Psychotherapy practice and research: repairing a strained alliance. American Psychological Association, 51, 100\%-16.

Goldfried, M.R., \& Wolfe, B.E. (1998). Toward a more clinically valid approach to therapy research. Journal of Consulting Clinical Psychological, 66, 143-50.

Goldstein, A.P., Heller, K., \& Sechrest, L.B. (1966). Psychotherapy and the Psychology of Behavior Change. New York: Wiley.

Goldstein, A.P. (1962). Patient-therapist Expectancies in Psychotherapy. Ney York: Pergamon Press.

Gottschalk, L.A., \& Auerbach, A.H. (1966). Methods of Research in Psychotherapy. New York: Appleton-Century-Crofts. 
Ricerca in Psicoterapia / Research in Psychotherapy 2010; 1(13): 92-119. http://www.researchinpsychotherapy.net

Greenberg, L.S., \& Pinsof, W. M. (1986). The Psychotherapentic Process: A Research Handbook. New York: Guilford Press.

Greenberg, L.S., \& Pinsof, W. M. (1986). Process Research: Current Trends and Future Perspectives. New York: Guilford Press.

Gurman, A.S., \& Razin, A.M. (1978). Effective Psychotherapy: A Handbook of Research. New York: Pergamon Press.

Harvey, J.H., \& Parks, M.M. (1982). Psychotherapy Research and Behavior Change. Washington, D.C: American Psychology.

Henry, W.P., Schacht, T.E., \& Strupp, H.H. (1986). Structural analysis of social behavior: Application to a study of interpersonal process in differential psychotherapeutic outcome. Journal of Consulting and Clinical Psychology, 54, 27-31.

Hersen, M., Michelson, L., \& Bellack, A.S. (1984). Issues in Psychotherapy Research. New York: Plenum.

Hill, C.E. (1989). Therapist Techniques and Client Outcomes. Eight Cases of Brief Psychotherapy. Newbury Park, CA: Sage.

Hill, C.E., Nutt, E.A., \& Jackson, S. (1994). Trends in Psychotherapy Process Research: Samples, Measures, Researchers, and Classic Publications. Journal of Counseling Psychology, 41, 364-37\%.

Hill, C.E. (2004). Methodological issues in studying psychotherapy processes and outcomes. In M.J. Lambert (Eds.), Bergin and Garfield's handbook of psychotherapy and behavior change (5th ed.) (pp. 84-135). New York: Wiley.

Hirsch, J.E. (2005). An index to quantify an individual's scientific research output. Proceedings of the National Academy of Sciences, 10, 216-269.

Horvath, A.O., \& Greenberg, L.S. (1994). The Working Alliance: Theory and Research. New York: Wiley.

Horwitz, L. (1974). Clinical Prediction in Psychotherapy. New York: Aronson.

Huber, W. (1987). Progress in Psychotherapy Research. Louvain-la-Neuve, Belgium: Presses Universitaires de Louvain.

Karasu, T.B. (1982). Psychotherapy Research: Methodological and Efficacy Issues. Washington, DC: American Psychiatric Press.

Kazdin, A.E. (1986). Comparative outcome studies of psychotherapy: methodological issues and strategies. Journal of Consulting and Clinical Psychology, 54, 95-105.

Kiesler, D.J. (1966). Some myths of psychotherapy research and the search for a paradigm. Psychological Bulletin, 65, 110-136.

Kiesler, D.J. (1971). Experimental design in psychotherapy research. In A. Bergin \& S. Garfield (Eds.), Handbook of Psychotherapy and Behavior Change. New York: John Wiley.

Kiesler, D.J. (1973). The Process of Psychotherapy: Empirical Foundation and System of Analysis, Chicago: Aldine.

Kiesler, D.J. (1981). The Process of Psychotherapy. Journal of Consulting and Clinical Psychology, 49, 212-215.

Lambert, M.J. (1979). The Effects of Psychotherapy. Montreal: Eden Press.

Lambert, M.J., Christensen, E.R., \& Dejulio, S.S. (1983). The Assessment of Psychotherapy Ontcome. New York: Wiley.

Lambert, J.M. (2007). Metodi ed obiettivi della ricerca in psicoterapia. In G. Nicolò \& S. Salvatore (Eds.), La ricerca sui risultati e sul processo in psicoterapia, (pp. 137-148), Roma: Carlo Amore. 
Ricerca in Psicoterapia / Research in Psychotherapy 2010; 1(13): 92-119. http://www.researchinpsychotherapy.net

Lambert, M.J., Bergin, A.E., \& Garfield, S.L. (2004). Introduction and Historical Overview. In H.L. Lennard \& A. Bernstein (Eds.). The Anatomy of Psychotherapy. New York: Columbia University Press.

Lesse, S. (1968). An Evaluation of the Results of the Psychotherapies. Journal of Neurological Neurosurg Psychiatry, 32, 1;1-1\%4.

Lietaer, G., Rombauts, J., \& Van Balen, R. (1990). Client-centered and Experiential Psychotherapy in the Nineties. Louvain-la-Neuve, Belgium: Presses Univ. de Louvain.

Lingiardi, V. (2006). La ricerca single-case. In N. Dazzi, V. Lingiardi \& A. Colli (Eds.), La ricerca in psicoterapia. Modelli e strumenti (pp. 123147). Milano: Raffaello Cortina.

Lis, A., Salcuni, S., \& Parolin, L. (2003). La valutazione empirica delle psicoterapie. Padova: Unipress.

Luborsky, L., Crits-Christoph, P., Mintz, J., \& Auerbach, A. (1988). Who Will Benefit from Psychotherapy? Predicting Therapentic Outcomes. New York: Basic Books.

Luborsky, L. (1984). Principles of Psychoanalytic Psychotherapy: A Manual for Supportive-Expressive Treatment. New York: Basic Books.

Luborsky, L. (197\%). Research cannot yet influence clinical practice. In A. Bergin \& H. Strupp (Eds.), Changing Frontiers in the Science of Psychotherapy (pp. 120-127). Chicago: Aldine.

Malan, D.H. (1976). Towards the Validation of Dynamic Psychotherapy. New York: Plenum.

Marmar, C.R. (1990). Psycotherapy Process Research: Progress, Dilemmas, and Future Direction. Journal Consulting and Clinical Psycology, 58, 265-272.

Marmor, J. (1974). The nature of the Psychotherapentic Process, in Psychiatry in Transiction: Selected papers of Judd Marmor. New York: Brunner/Mazzel.

Meltzoff, J., \& Kornreich, M. (1970). Research in Psychotherapy. New York: Atherton.

Migone, P. (2006). Breve storia della ricerca in psicoterapia: Con una nota sui contributi italiani. In N. Dazzi, V. Lingiardi \& , A. Colli (Eds.), La ricerca in psicoterapia. Modelli e strumenti (pp. 31-48). Milano: Cortina.

Migone, P. (1996). La ricerca in psicoterapia: storia, principali gruppi di lavoro, stato attuale degli studi sul risultato e sul processo. Rivista Sperimentale di Freniatria, 2, 182-238.

Miller, N., Luborsky, L., Barber, J.P., \& Docherty, J. (1993). Psychodynamic Treatment Research: A Handbook for Clinical Practice. New York: Basic Books.

Molenaar, P.C..M., \& Valsiner, J. (2005). How Generalization Works through the Single Case: A Simple Idiographic Process Analysis of an Individual Psychotherapy. International Journal of Idiographic Science, retrivial from: www.valsiner.com

Morrow-Bradley, C., \& Elliott, R. (1986). The utilization of psychotherapy research by practicing psychotherapists. American Psychological Association, 41, 188-9\%.

Mowrer, O.H. (1953). Psychotherapy: Theory and Research. New York: Ronald.

Nicolò, G., \& Salvatore, S. (2007). La ricerca sui risultati e sul processo in psicoterapia. Roma: Carlo Amore. 
Ricerca in Psicoterapia / Research in Psychotherapy 2010; 1(13): 92-119. http://www.researchinpsychotherapy.net

Orlinsky, D.E., Grawe, K., \& Parks, B.K. (1994). Process and outcome in psychotherapy. In A. Bergin \& S. Garfield (Eds.), Handbook of Psychotherapy and Behavior Change, (4th ed.) (pp. 270-376). New York: Wiley.

Orlinsky, D.E., \& Howard, K.I. (1967). The good Therapy hour. Archives of General Psychiatry, 16, 621-632.

Orlinsky, D.E., \& Howard, K.I. (1978). The relation of process to outcome in psychotherapy. In A. Bergin \& S. Garfield's (Eds.). Handbook of Psychotherapy and Behavior Change, (2th ed.). New York: Wiley.

Orlinsky, D.E., Howard, K.I. (1986). Process and outcome in psychotherapy. In A. Bergin \& S. Garfields (Eds.), Handbook of Psychotherapy and Behavior Change (3th ed.). New York: Wiley.

Orlinsky, D.E., Howard, K.I. (1987). A generic model of psychotherapy. Journal of Integrative and Eclectic Psychology, 6, 6-2\%.

Orlinsky, D.E., Rønnestad, M.H, Willutzki, U. (2004). Fifty years of psychotherapy process-outcome research: Continuity and Change. In A. Bergin \& S. Garfields (Eds.), Handbook of Psychotherapy and Behavior Change (5th ed.). New York:Wiley.

Orlinsky, D.E., \& Howard, K.I. (1975). Varieties of Psychotherapentic Experience. Multivariate analyses of patients' and therapists' reports. New York: Teachers College.

Orlinsky, D.E., \& Russell, R.L. (1994). Tradition and change in psychotherapy process-outcome research: Notes on the fourth generation. In R.L. Russell (Eds.), Reassessing Psychotherapy Research (pp. 185214). New York: Guilford.

Pachankis, J.E., \& Goldfried, M.R. (200\%). On the nex generation of process research. Clinical Psychology Review, 27, 760-768.

Parloff, M.B. (1979). Can psychotherapy research guide the policymaker? A little knowledge may be a dangerous thing. American Psychologist, 34, 296-306.

Popolo, R., \& Semerari, A. (2007). La ricerca empirica in psicoterapia. In G. Nicolò \& S. Salvatore (Eds.), La ricerca sui risultati e sul processo in psicoterapia (pp. 93-108). Roma: Carlo Amore.

Eysenck, H.J. (1952). The effects of psychotherapy: An evaluation. Journal of Consulting Psychology, 16, 319-324.

Rice, L.N., \& Greenberg, L.S. (1984). Patterns of Change: Intensive Analysis of Psychotherapy Process. New York: Guilford.

Rogers, C.R., \& Dymond, R.F. (1954). Psychotherapy and Personality Change. Chicago: University of Chicago Press.

Rogers, C.R., Gendlin, E.T., Kiesler, D., \& Truax, C.B. (1967). The Therapentic Relationship and its Impact. Madison: University of Wisconsin Press.

Rubinstein, E.A., \& Parloff, M.B. (1962). Research in Psychotherapy. (Vol. 1). Washington, DC: American Psychological Association.

Russell, R.L., \& Wandrei M.L. (1996). Narrative and the process of psychotherapy: Theoretical foundations and empirical support. In $\mathbf{H}$. Rosen, \& K.T. Kuehlwein (Eds.), Constructing realities (pp. 307-336). San Francisco: Jossey-Bass.

Russell, R.L. (1987). Language in Psychotherapy. New York: Plenum.

Russell, R.L., \& Staszewski, C. (1988). The unit problem: some systematic distinctions and critical dilemmas for psychotherapy process research. Psychotherapy, 25, 191-200. 
Ricerca in Psicoterapia / Research in Psychotherapy 2010; 1(13): 92-119. http://www.researchinpsychotherapy.net

Russell, R.L., \& Orlinsky, D. (1996). Psychotherapy Research in Historical Perspective: Some Implications for Mental Health Care Policy. Archives of General Psychiatry, 53, 708-713.

Russell, R.R., \& Trull, T.J. (1986). Sequential analyses of language variables in psychotherapy process research. Journal of Consulting and Clinical Psychology, 54, 16-21.

Russell, R.L (1994). Reassessing Psychotherapy Research, New York: Guilford.

Salvatore, S., \& Nicolò, G. (2005). La ricerca in psicoterapia: Materiali di studio e prospettive dal contesto italiano. Roma: Carlo Amore.

Salvatore, S., Mossi, P.G., \& Gennaro, A. (2007). Il discorso sulla psicoterapia, tra dinamiche istituzionali,concezioni della mente, modelli di intervento, pratiche di ricerca. In G. Nicolò \& S. Salvatore (Eds.), La ricerca sui risultati e sul processo in psicoterapia (pp. 43-66). Roma: Carlo Amore.

Scheflen, A.E. (1973). Communicational Structure: Analysis of a Psychotherapy Transaction. Bloomington, IN: Indiana University Press.

Shlien, J.M., Hunt, H.F., Matarazzo, J.D., \& Savage, C. (1966). Research in Psychotherapy (Vol. 3). Washington, DC: American Psychological Association.

Shoham-Salomon, V. (1990). Interrelating research processes of process research. Journal of Consulting and Clinical Psychology, 58, 295-303.

Slife, B.D. (2004). Theoretical Challenges to Therapy Practice and Research: The Constraint of Naturalism. In J.M. Lambert (Ed.). Bergin and Garfield's Handbook of Psychotherapy and Behavior Change (pp. 44-83). New York: Wiley and Sons.

Smith, M.L., Glass, G.V., \& Miller, T.I. (1980). The Benefits of Psychotherapy. Baltimore, MD: Johns Hopkins University Press.

Snyder, W.U. (1961). The Psychotherapy Relationship. New York: Macmillian.

Spitzer, R.L., \& Klein, R.F. (1976). Evaluation of Psychological Therapies: Psychotherapies, Behavior Therapies, Drug Therapies, and Interactions. Baltimore, MD: Johns Hopkins University Press.

Stieper, D.R., \& Wiener, D.N. (1965). Dimensions of Psychotherapy. Chicago: Aldine.

Stiles, W.B. (1988). Psychotherapy process-outcome correlations may be misleading. Psychotherapy, 25, 27-35.

Stiles, W.B., \& Shapiro, D.A., \& Elliott, R. (1986). Are all psychotherapies equivalent? American Psychologist, 41, 165-180.

Stricker, G., \& Gold, J.R. (1993) Comprehensive handbook of psychotherapy integration. New York: Plenum.

Strupp, H.H., \& Luborsky, L. (1962). Research in Psychotherapy (Vol. 2). Washington, DC: American Psychological Association.

Strupp, H.H. (1973). Psychotherapy: Clinical, Research, and Theoretical Issues. New York: Grune \& Stratton.

Strupp, H. (1997). Research, practice, and managed care. Psychotherapy, 34, 91.94.

Strupp, H.H., Fox, R.E., \& Lessler, K. (1966). Patients View their Psychotherapy. Baltimore, MD: Johns Hopkins University Press. University Press. 
Ricerca in Psicoterapia / Research in Psychotherapy 2010; 1(13): 92-119. http://www.researchinpsychotherapy.net

Strupp, H.H., Hadley, S.W., \& Gomes-Schwartz, B. (1978). Psychotherapy for Better or Worse: The Problem of Negative Effects. New York: Aronson.

Strupp, H.H. (1960). Psychotherapists in Action. New York: Basic Books.

Talley, F., Butler, S., \& Strupp, H.H. (1994). Research Findings and Clinical Practice: Bridging the Chasm. New York: Basic Books.

Terence, J., \& Tracey G. (2003). Concept Mapping of Therapentic Common Factors. Psychotherapy Research, 13, 401- 413.

Toukmanian, S.G., \& Rennie, D.L. (1992). Psychotherapy Process Research: Paradigmatic and Narrative Approaches. Newbury Park, CA: Sage.

van Raan, A.F.J., Visser, M.S., van Leeuwen, T.N., \& van Wijk, E. (2003). Bibliometric analysis of psychotherapy research: Performance assessment and position in the journal landscape. Psychotherapy Research, 13, 511-528.

Virgil, D. (1994). Dictionary of bibliometrics. New York: The Haworth Press.

Volsky, T., Magoon, T.M., Norman, W.T. \& Hoyt, D.P. (1965). The Outcomes of Counseling and Psychotherapy. Minneapolis: University of Minnesota Press.

Waskow, I.E., \& Parloff, M.B. (1975). Psychotherapy Change Measures. Rockville, MD: National Institute of Mental Health.

Weiss, D.W., Marmar, C.R., \& Horowitz, M.J. (1988). Do the ways in which psychotherapy process ratings are made make a difference? The effects of mode of presentation, segment, and rating format on interrater reliability. Psychotherapy: Theory, Research, Practice, and Training, 25, 44-50.

Westen, D., \& Weinberger, J. (2004). When clinical description becomes statistical prediction. American Psychologist, 59, 595-613.

Westen, D., Morrison, K., \& Thompson-Brenner, H. (2004). The empirical status of empirically supported psychotherapies: assumptions, findings, and reporting in controlled clinical trials. Psychological Bullettin, 130, $631-663$.

Wexler, D.A., \& Rice, L.N. (1974). Innovations in Client-Centered Therapy. New York: Wiley.

White, M., \& Epston, D. (1990). Narrative means to therapeutic ends. New York: Norton and Company.

Widlocher, D. (1994). A case is not a fact. International Journal of Psychoanalysis, 75, 1233-1244.

Williams, J.B.W., \& Spitzer, R.L. (1984). Psychotherapy Research: Where are we and where should we go?. New York: Guilford.

Wolberg, L. (1978). The technique of psychotherapy. New York: Grune \& Stratton.

Wolff, W., \& Precker, P.A. (1952). Success in Psychotherapy. New York: Grune \& Stratton. 
Ricerca in Psicoterapia / Research in Psychotherapy 2010; 1(13): 92-119. http://www.researchinpsychotherapy.net

The Psychotherapy Process Research from 1998 to 2007: An early step toward a critical review

Abstract: This preliminary survey wants to be a first contribution to a critical review on psychotherapy process research. The aim is to describe: how can process research be divided? According to what dimension? In how many ways? The goals of this research are to trace the implicit and basic dimensions that can organize the heterogeneity of this sector in a consistent way. According to our goal we used a grid built up through several variables by means of which we segmented the studies on process research (both process and process-outcome studies) extracted from 13 scientific journals over the last ten years (1998-200\%). The goals were: a) Quantifying and classifying the scientific production of the last ten years b) exploring the main models that articulate and organize the field. We found two main epistemological models in the process research: the model of analysis and the interpretative model. These two models give rise to three different approaches: Conjectural approach, Sperimental and Mixed Approach, both rising questions about the construct of significance, the choice of the objects of analysis and the relation between the object of the research and the methodology adopted.

Key-words: Critical review; psychotherapy process research; process-outcome research. 
Ricerca in Psicoterapia / Research in Psychotherapy 2010; 1(13): 92-119. http://www.researchinpsychotherapy.net

\section{Appendice A}

\section{Popolazione}

American Journal of Psychotherapy; Annals of the American Psychotherapy Assn, Annual Review of Clinical Psychology; Archives of Psychiatry and Psychotherapy; Australasian Journal of Psychotherapy; Behavioural and Cognitive Psychotherapy; Body; Movement and Dance in Psychotherapy; British Journal of Clinical Psychology; British Journal of Psychotherapy; Chinese Journal of Clinical Psychology; Clinical Psychology \& Psychotherapy; Clinical Psychology Review; Clinical Psychology: Science and Practice; Counselling \& Psychotherapy Research; European Journal of Psychotherapy and Counselling; International Journal of Group Psychotherapy; International Journal of Psychotherapy; Journal of Child Psychotherapy; Journal of Clinical Psychology; Journal of Clinical Psychology in Medical Settings; Journal of Cognitive Psychotherapy; Journal of College Student Psychotherapy; Journal of Consulting and Clinical Psychology; Journal of Contemporary Psychotherapy; Journal of Family Psychotherapy; Journal of Gay \& Lesbian Psychotherapy; Journal of Group Psychotherapy, Psychodrama \& Sociometry; Journal of Infant, Child \& Adolescent Psychotherapy; Journal of Psychotherapy Integration; Journal of Social \& Clinical Psychology; Pakistan Journal of Social and Clinical Psychology; Pragmatic Case Studies in Psychotherapy; Psychoanalytic Psychotherapy; Psycho-analytic Psychotherapy in South Africa; Psychology and Psychotherapy: Theory, Research and Practice; Psychotherapy and Psychosomatics; Psychotherapy Research; Psychotherapy: Theory, Research, Practice, Training; The Arts in Psychotherapy.

\section{Abbreviazioni}

$\begin{array}{ll}\text { AJP } & \text { American journal of psychotherapy } \\ \text { CPP } & \text { Clinical psychology and psychotherapy } \\ \text { CPSP } & \text { Clinical psychology: science and practice } \\ \text { CPR } & \text { Counselling \& psychotherapy research } \\ \text { IJGP } & \text { International journal of group psychotherapy } \\ \text { JCP } & \text { Journal of clinical psychology } \\ \text { JCCP } & \text { Journal of consulting and clinical psychology } \\ \text { JFP } & \text { Journal of family psychotherapy } \\ \text { JPI } & \text { Journal of psychotherapy integration } \\ \text { PCSP } & \text { Pragmatic case studies in psychotherapy } \\ \text { PAPTRAP } & \text { Psychology and psychotherapy: theory, research and practice } \\ \text { PR } & \text { Psychotherapy research } \\ \text { PTRPT } & \text { Psychotherapy: teory, research, practice training }\end{array}$


Ricerca in Psicoterapia / Research in Psychotherapy 2010; 1(13): 92-119. http://www.researchinpsychotherapy.net

\section{Appendice B}

Autovalori e inerzia ricalcolata con la formula di Benzecrì.

\begin{tabular}{|c|c|c|c|c|}
\hline NUMERO & VALEUR & VARIANZA & VAR CUMUL & VAR RICAL Benzecrì \\
\hline 1 & 0,3889 & 9,3 & 9,3 & 32,08788986 \\
\hline 2 & $\mathbf{0}, 28$ & 6,7 & 16 & 48,04141966 \\
\hline 3 & 0,1919 & 4,59 & 20,59 & 55,02586633 \\
\hline 4 & 0,164 & 3,92 & 24,51 & 59,93142188 \\
\hline 5 & 0,1476 & 3,53 & 28,04 & 63,78591882 \\
\hline 6 & 0,1391 & 3,33 & 31,37 & $6 \%, 14545862$ \\
\hline 8 & 0,1254 & 3 & 34,37 & 69,77880466 \\
\hline 8 & 0,1225 & 2,93 & 37,3 & 72,26975766 \\
\hline 9 & 0,1217 & 2,91 & 40,21 & 74,72212627 \\
\hline 10 & 0,1187 & 2,84 & 43,05 & $78,032485 \% 2$ \\
\hline 11 & 0,1131 & $2, \%$ & 45,75 & 79,0890929 \\
\hline 12 & 0,1091 & 2,61 & 48,36 & 80,97348355 \\
\hline 13 & $0,10 \%$ & 2,56 & 50,92 & 82,77047456 \\
\hline 14 & 0,1008 & 2,41 & 53,33 & 84,32153652 \\
\hline 15 & 0,0969 & 2,32 & 55,65 & 85,7271692 \\
\hline 16 & 0,0951 & 2,27 & 57,92 & 87,06809472 \\
\hline 17 & 0,0929 & 2,22 & 60,15 & 88,33200427 \\
\hline 18 & 0,0906 & 2,17 & 62,31 & 89,51783236 \\
\hline 19 & 0,0896 & 2,14 & 64,46 & 90,67048843 \\
\hline
\end{tabular}

